\title{
Using technology to deliver cancer follow-up: a systematic review
}

\author{
Rebekah Dickinson ${ }^{1}$, Susan Hall ${ }^{2 *}$, Jenny E Sinclair ${ }^{2}$, Christine Bond ${ }^{2}$ and Peter Murchie ${ }^{2}$
}

\begin{abstract}
Background: People with cancer receive regular structured follow up after initial treatment, usually by a specialist in a cancer centre. Increasing numbers of cancer survivors prompts interest in alternative structured follow-up models. There is worldwide evidence of increasing interest in delivering cancer follow-up using technology. This review sough evidence supporting the use of technology in cancer follow-up from good quality randomised controlled trials.
\end{abstract}

Method: A search strategy was developed to identify randomised controlled trials and reviews of randomised trials of interventions delivering some aspect of structured cancer follow-up using new technologies. Databases searched were: All EBM Reviews; Embase; Medline (No Revisions); Medline (Non-Indexed Citations), and CAB Abstracts. Included articles were published in English between 2000 and 2014. Key words were generated by the research question. Papers were read independently and appraised using a standardised checklist by two researchers, with differences being resolved by consensus [J Epidemiol Community Health, 52:377-384, 1998]. Information was collected on the purpose, process, results and limitations of each study. All outcomes were considered, but particular attention paid to areas under consideration in the review question.

Results: The search strategy generated 22879 titles. Following removal of duplicates and abstract review 17 full papers pertaining to 13 randomised controlled studies were reviewed. Studies varied in technologies used and the elements of follow-up delivered, length of follow-up, tumour type and numbers participating. Most studies employed only standard telephone follow-up. Most studies involved women with breast cancer and included telephone follow-up. Together the results suggest that interventions comprising technology had not compromised patient satisfaction or safety, as measured by symptoms, health related quality of life or psychological distress. There was insufficient evidence to comment on the cost effectiveness of technological cancer follow-up interventions.

Conclusions: Modern technology could deliver cancer follow-up that is acceptable and safe. More research is required to develop cancer follow-up systems which exploit modern technology, which should be assessed using randomised trials, with consistent outcomes, so that evidence on the acceptability, safety, cost effectiveness and impact in quality of life of technological follow-up can accumulate and be made available to patients, professionals and policy makers.

\section{Background}

Following the completion of their primary treatment for cancer most patients enter a programme of structured follow-up [1,2]. This is usually based in secondary care and involves regular face to face consultations with specialist cancer doctors; the precise frequency and content of follow-up visits varies according to cancer site and local

\footnotetext{
* Correspondence: s.hall@abdn.ac.uk

${ }^{2}$ Division of Applied Health Science, Centre of Academic Primary Care, University of Aberdeen, Polwarth Building, Foresterhill, Aberdeen AB25 2ZD, Scotland, UK

Full list of author information is available at the end of the article
}

and national guidelines [3]. Follow-up care is generally focused on detecting recurrent disease, monitoring the effects of treatment and providing ongoing support to patients and their families and there is good evidence that such care is valued by patients [4].

Current models of cancer follow-up are likely to be unsustainable due to two important factors. Firstly, as the population ages and treatment improves cancer prevalence increases year on year [5]. This means that secondary care services are tasked with the delivery of follow-up to an increasing number of patients, and generally without corresponding increase in resources [6]. Secondly, accessing 
aftercare can be problematic for certain patient groups, especially those who live in remote and rural areas distant from cancer centres [3]. Access difficulties to cancer follow-up care could be one reason for the observation in some areas of poorer outcomes in rural, compared to urban, cancer patients [7]. In contrast modern technology develops apace and offers increasing capability and functionality to patients, professionals and health systems for care delivery. Furthermore, the current population of cancer patients are increasingly familiar with technology and consuming healthcare information and services on digital platforms.

These issues together are the drivers to develop modern and alternative models of cancer follow-up. To date these have included varying the person delivering care (e.g. a specialist nurse rather than a doctor) and varying the location of cancer follow-up delivery (e.g. primary rather than secondary care) [4]. Models of care that have been subjected to randomised trials have included shared care, nurse-led follow-up and GP-led follow-up, as well as shifting the locus of care from hospital to the community [4]. A further alternative is to exploit the current innovative technological environment and seek to understand how digital means may be employed to deliver some or all aspects of cancer follow-up care [8].

"Telemedicine" is defined as using technology to share information over a distance between healthcare providers (e.g. between GPs and hospital specialists), whereas "telehealthcare" is defined as using technology to provide personalised healthcare to patients at a distance $[9,10]$. To date some investigators have incorporated the use of land-line and mobile telephones into the delivery of cancer follow-up [11-13].

As technology improves and cancer prevalence increases interest in developing models of follow-up care that employ novel technologies is certain to increase, for example monitoring chemotherapy effects using smartphones [12]. This systematic review was conducted to evaluate existing evidence on the clinical safety, patient acceptability, cost effectiveness and impact on quality of life from telemedicine and telehealthcare where it has been applied to cancer follow-up.

\section{Methods}

\section{Search and identification of studies}

The population of interest was adults with cancer. The intervention was cancer follow up using technology and the control usual care. Inclusion criteria were randomised controlled studies published in English between 2000 and 2014, whose intervention included a telemedicine or telehealthcare element in the intervention. Studies not meeting these criteria were excluded. The key outcomes of interest were patient acceptability (satisfaction), clinical safety and cost effectiveness.
A search strategy based on key words to reflect the review aim was designed in conjunction with a medical librarian and is included as Additional file 1.

The searches were run in February 2014 on the following databases: All EBM Reviews; Embase; Medline (No Revisions); Medline (Non-Indexed Citations); CAB Abstracts. Retrieved citations were exported to Refworks (www.refworks.com). All identified titles were read and those not meeting the inclusion criteria were excluded, as were duplicates. Abstracts of the remaining studies were screened against the inclusion criteria and full articles were then retrieved.

\section{Data collection}

Critical appraisal of selected studies was undertaken using a standardized checklist [14]. This was done by two researchers (RD and $\mathrm{SH}$ ). There was subsequent discussion of assigned scores, and discussion and resolution of any differences by consensus. Papers were analyzed thematically considering particularly outcomes that related to themes highlighted in the review question, namely, clinical safety, patient acceptability, cost effectiveness and impact on quality of life. Clinical safety was defined as any outcome related to recurrent cancer or mortality. Patient acceptability was defined as outcomes reflecting how easily patients had found engaging with an intervention, the extent to which it met their healthcare needs, or how it impacted upon their satisfaction with services. Cost effectiveness related to the reporting of appropriate economic data. Quality of life related to any outcomes reflecting symptoms or accepted or validated measures of health related quality of life. Information was collected on the purpose, process, results and limitations of each study using a standardised data collection template. All outcomes were considered with particular attention paid to issues of patient acceptability and satisfaction, clinical safety, cost and impact on quality of life. Where quantitative data was presented it was tabulated as $p$ values, confidence intervals and effect sizes. A narrative analysis of all papers was also conducted to identify the emergent common and contrasting themes from the reported studies.

\section{Results \\ Study selection}

Figure 1, a PRISMA diagram, displays the data for the number of titles initially identified, then excluded along with duplicates and the final number of randomised studies identified and included.

\section{Characteristics of included studies}

Seventeen papers pertaining to 13 randomised studies were included in the review. Tables 1 and 2 considers and displays each of these studies in detail including terms of 


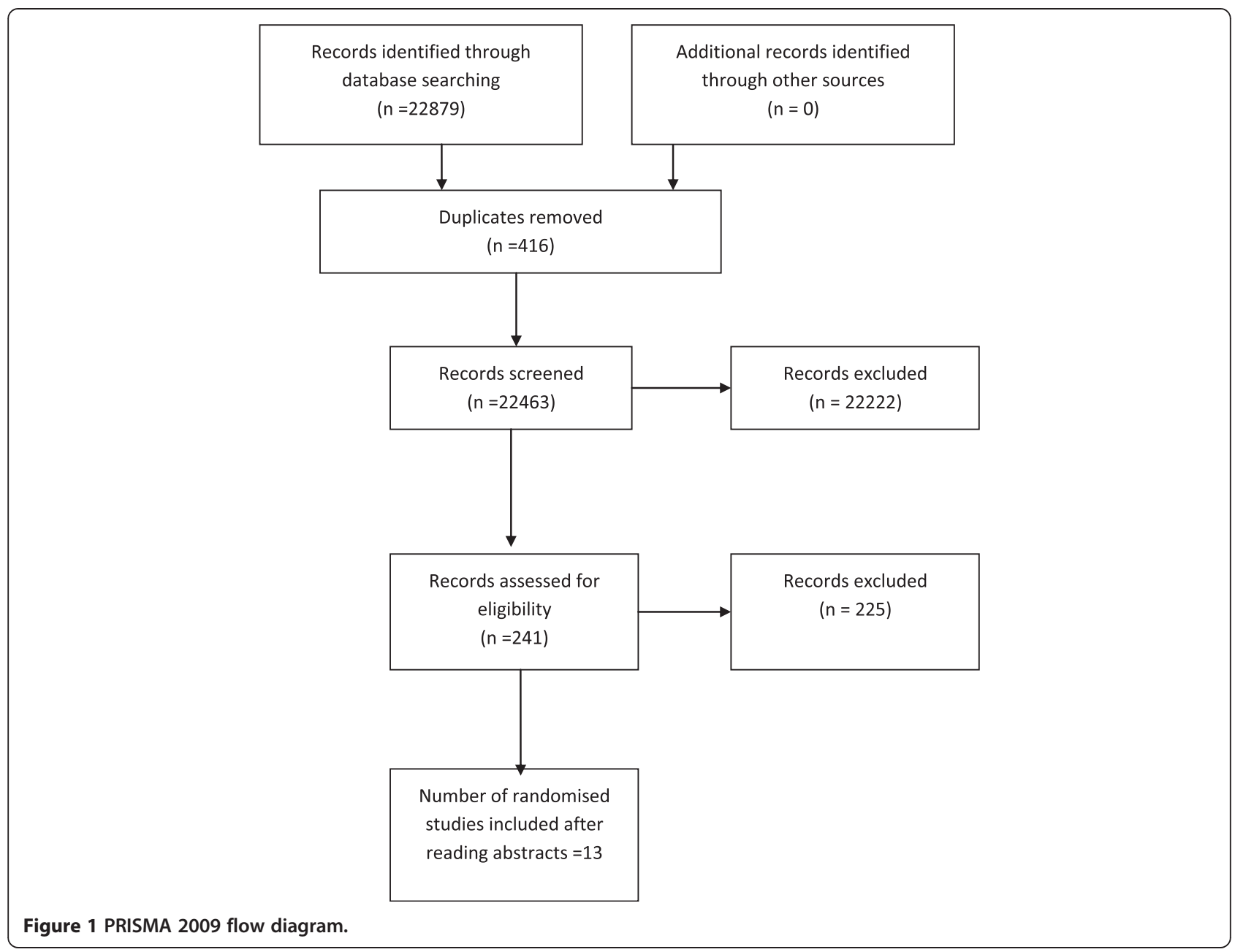

patient population, intervention, outcome measures, key results, study quality score, measures used and detailed results including effect sizes.

\section{Included patients and countries}

Of the thirteen included studies eight had been conducted amongst women with breast cancer; one amongst men with prostate cancer and one amongst people with colorectal cancer [11,13,15-21]. Of the remaining three, a Scottish study using mobile phones to monitor symptoms was conducted in patients with breast, lung or colorectal cancer currently undergoing chemotherapy, a US study included patients with any solid tumour or non-Hodgkin's lymphoma who were currently undergoing chemotherapy and a Korean study of cancer survivors who had reported severe fatigue $[12,22-24]$. One study was conducted in the Netherlands [17], one in Australia [21], two each in the UK $[11,12,25]$, and Canada [15,20], six in the USA [16,18,19,22,23,26] and one in Korea [24]. The age ranges of patients were not consistently recorded.

\section{Synthesis of key outcomes}

\section{Range of interventions and technologies employed}

Of the 13 randomised studies the majority, seven, of the interventions were relatively low-tech and had simply employed standard telephone calls to cancer aftercare recipients in their own homes, as an alternative to standard follow-up [11,13,16-19,21]. These calls were generally delivered by specialist nurses. The content, duration and frequency varied across studies. Calls were mainly scheduled, regular, and lasted approximately 30 minutes, and delivered components of symptom monitoring, information sharing, and emotional support. Two further interventions employed remote symptom monitoring using a smartphone/personal digital assistant (PDA) $[12,20]$. A further intervention employed an automated voice activated telephone response system to monitor symptom severity $[22,23]$. One intervention comprised a computer programme, completed by those in aftercare, which provided the patient with information and assisted decision making [15]. Participants were encouraged to work through the computer programme prior to their face 
Table 1 Details of included studies including critical appraisal scores

\begin{tabular}{|c|c|c|c|c|c|c|c|}
\hline Study and location & $\begin{array}{l}\text { Patient } \\
\text { population }\end{array}$ & Intervention & Control & $\begin{array}{l}\text { Length of } \\
\text { follow up }\end{array}$ & Study outcomes & Results & $\begin{array}{c}\text { Critical } \\
\text { appraisal score }\end{array}$ \\
\hline \multirow{5}{*}{$\begin{array}{l}\text { Beaver et al., } 2009 \\
\text { Manchester, UK [11] }\end{array}$} & \multirow{5}{*}{$\begin{array}{l}374 \text { breast } \\
\text { cancer patients }\end{array}$} & \multirow{5}{*}{$\begin{array}{l}\text { Telephone follow up } \\
\text { by specialist nurses }\end{array}$} & \multirow[t]{5}{*}{ Usual hospital care } & \multirow{5}{*}{$\begin{array}{l}24 \text { months } \\
\text { (mean) }\end{array}$} & Psychological morbidity & \multirow{5}{*}{$\begin{array}{l}\text { Equivalence trial - : no } \\
\text { difference between the } \\
\text { two groups }\end{array}$} & Study Quality - 8/10 \\
\hline & & & & & $\begin{array}{l}\text { Participant's needs for } \\
\text { information }\end{array}$ & & External validity $-2 / 3$ \\
\hline & & & & & Participant's satisfaction & & $\begin{array}{l}\text { Internal Validity } \\
\text { (bias) }-6 / 7\end{array}$ \\
\hline & & & & & $\begin{array}{l}\text { Clinical Investigations } \\
\text { ordered }\end{array}$ & & $\begin{array}{c}\text { Internal Validity } \\
\text { (selection bias) }-6 / 6\end{array}$ \\
\hline & & & & & $\begin{array}{l}\text { Time to detection of } \\
\text { recurrent disease }\end{array}$ & & $\begin{array}{l}\text { Power - 1/1 } \\
\text { (Total - 23/27) }\end{array}$ \\
\hline \multirow{2}{*}{$\begin{array}{l}\text { Beaver et al., } 2009 \\
\text { (Economic evaluation) } \\
\text { Manchester, UK [25] }\end{array}$} & \multirow{2}{*}{$\begin{array}{l}374 \text { breast } \\
\text { cancer patients }\end{array}$} & \multirow{2}{*}{$\begin{array}{l}\text { Cost minimization } \\
\text { analysis of RCT above }\end{array}$} & \multirow[t]{2}{*}{-} & \multirow{2}{*}{$\begin{array}{l}24 \text { months } \\
\text { (mean) }\end{array}$} & Primary: NHS resource use & \multirow{2}{*}{$\begin{array}{c}\text { Telephone follow-up more } \\
\text { costly (mean difference } £ 55 \\
\text { but telephone patients had } \\
\text { lower personal costs (mean } \\
\text { difference } £ 47 \text { ) }\end{array}$} & \multirow{2}{*}{$\begin{array}{l}\text { No score as cost } \\
\text { analysis }\end{array}$} \\
\hline & & & & & $\begin{array}{l}\text { Secondary: patient, carer } \\
\text { and productivity courses }\end{array}$ & & \\
\hline \multirow[t]{5}{*}{$\begin{array}{l}\text { Davison and Degner, } \\
2002 \text { Vancouver, } \\
\text { Canada [15] }\end{array}$} & \multirow[t]{5}{*}{$\begin{array}{l}749 \text { breast } \\
\text { cancer patients }\end{array}$} & \multirow[t]{5}{*}{$\begin{array}{l}\text { Computer programme } \\
\text { providing information and } \\
\text { assisting decision making }\end{array}$} & \multirow[t]{5}{*}{$\begin{array}{l}\text { Standard care only- asked } \\
\text { about decision making } \\
\text { before clinic appointment }\end{array}$} & \multirow[t]{5}{*}{ One clinic visit } & $\begin{array}{l}\text { Involvement in decision } \\
\text { making }\end{array}$ & $\begin{array}{l}\text { Women in the intervention } \\
\text { group reporting playing a } \\
\text { more passive role. }\end{array}$ & Study Quality - 6/10 \\
\hline & & & & & \multirow[t]{4}{*}{ Patient satisfaction } & \multirow{4}{*}{$\begin{array}{l}\text { Patient satisfaction was } \\
\text { high in both groups }\end{array}$} & External validity $-2 / 3$ \\
\hline & & & & & & & $\begin{array}{l}\text { Internal Validity } \\
\text { (bias) }-5 / 7\end{array}$ \\
\hline & & & & & & & $\begin{array}{c}\text { Internal Validity } \\
\text { (selection bias) }-4 / 6\end{array}$ \\
\hline & & & & & & & $\begin{array}{l}\text { Power - 0/1 } \\
\text { (Total - 17/27) }\end{array}$ \\
\hline \multirow[t]{5}{*}{$\begin{array}{l}\text { Harrison et al., } 2011 \\
\text { Sydney, Australia [21] }\end{array}$} & \multirow[t]{5}{*}{$\begin{array}{l}75 \text { patients with } \\
\text { colorectal cancer }\end{array}$} & \multirow[t]{5}{*}{$\begin{array}{l}5 \text { telephone calls from a } \\
\text { specialist colorectal nurse } \\
\text { in } 6 \text { months after discharge }\end{array}$} & \multirow[t]{5}{*}{ Standard care } & \multirow[t]{5}{*}{6 months } & $\begin{array}{l}\text { Unmet supportive care } \\
\text { needs }\end{array}$ & $\begin{array}{l}\text { No difference between the } \\
\text { groups for unmet needs and } \\
\text { health service utilization }\end{array}$ & Study Quality - 8/10 \\
\hline & & & & & Health service utilization & \multirow{4}{*}{$\begin{array}{c}\text { Quality of life scores higher } \\
\text { in the intervention group at } \\
6 \text { months }\end{array}$} & External validity $-2 / 3$ \\
\hline & & & & & Quality of life & & $\begin{array}{l}\text { Internal Validity } \\
\text { (bias) }-5 / 7\end{array}$ \\
\hline & & & & & & & $\begin{array}{c}\text { Internal Validity } \\
\text { (selection bias) }-6 / 6\end{array}$ \\
\hline & & & & & & & $\begin{array}{c}\text { Power - 0/1 } \\
\text { (Total - 21/27) }\end{array}$ \\
\hline
\end{tabular}


Table 1 Details of included studies including critical appraisal scores (Continued)

\begin{tabular}{|c|c|c|c|c|c|c|c|}
\hline \multirow{5}{*}{$\begin{array}{l}\text { Hegel et al., } 2010 \\
\text { New Hampshire, } \\
\text { USA [16] }\end{array}$} & \multirow[t]{5}{*}{$\begin{array}{c}31 \text { Breast } \\
\text { cancer patients }\end{array}$} & \multirow{5}{*}{$\begin{array}{l}6 \text { weekly session of } \\
\text { telephone delivered } \\
\text { problem solving } \\
\text { occupational therapy }\end{array}$} & \multirow[t]{5}{*}{ Usual care } & \multirow[t]{5}{*}{12 weeks } & \multirow{5}{*}{$\begin{array}{l}\text { Primary outcome: feasibility } \\
\text { of conducting the trial } \\
\text { Secondary outcomes: } \\
\text { functional, quality of life } \\
\text { and emotional status }\end{array}$} & \multirow[t]{5}{*}{ Overall positive outcomes } & Study Quality - 8/10 \\
\hline & & & & & & & External validity $-3 / 3$ \\
\hline & & & & & & & $\begin{array}{l}\text { Internal Validity } \\
\text { (bias) }-5 / 7\end{array}$ \\
\hline & & & & & & & $\begin{array}{c}\text { Internal Validity } \\
\text { (selection bias) }-6 / 6\end{array}$ \\
\hline & & & & & & & $\begin{array}{l}\text { Power - 0/1 } \\
\text { (Total - 20/20) }\end{array}$ \\
\hline \multirow{5}{*}{$\begin{array}{l}\text { Kearney et al., } 2008 \\
\text { Stirling, Scotland [12] }\end{array}$} & \multirow{5}{*}{$\begin{array}{l}112 \text { cancer } \\
\text { patients }\end{array}$} & \multirow{5}{*}{$\begin{array}{l}\text { Mobile phone-based } \\
\text { remote monitoring } \\
\text { during chemotherapy }\end{array}$} & \multirow[t]{5}{*}{ Standard care } & \multirow[t]{5}{*}{16 weeks } & \multirow{5}{*}{$\begin{array}{l}\text { Chemotherapy related } \\
\text { morbidity - } 6 \text { common } \\
\text { symptoms, nausea, vomiting, } \\
\text { fatigue, mucositis, hand-foot } \\
\text { syndrome and diarrhoea }\end{array}$} & \multirow{5}{*}{$\begin{array}{l}\text { Higher reports of fatigue } \\
\text { in the control group and } \\
\text { lower reports of hand-foot } \\
\text { syndrome in the control } \\
\text { group }\end{array}$} & Study Quality - 8/10 \\
\hline & & & & & & & External validity $-1 / 3$ \\
\hline & & & & & & & $\begin{array}{l}\text { Internal Validity } \\
\text { (bias) }-5 / 7\end{array}$ \\
\hline & & & & & & & $\begin{array}{c}\text { Internal Validity } \\
\text { (selection bias) }-6 / 6\end{array}$ \\
\hline & & & & & & & $\begin{array}{l}\text { Power - 0/1 } \\
\text { (Total - 20/27) }\end{array}$ \\
\hline \multirow{5}{*}{$\begin{array}{l}\text { Kimman et al., } \\
2011 \text { Maastricht, } \\
\text { Netherlands [17] }\end{array}$} & \multirow{5}{*}{$\begin{array}{l}299 \text { women } \\
\text { with breast } \\
\text { cancer }\end{array}$} & $\begin{array}{l}\text { Nurse led telephone } \\
\text { follow up or }\end{array}$ & \multirow{5}{*}{$\begin{array}{l}\text { Hospital follow up or } \\
\text { hospital follow up } \\
\text { plus EGP }\end{array}$} & \multirow[t]{5}{*}{18 months } & $\begin{array}{l}\text { Health related quality of } \\
\text { life (HRQoL) }\end{array}$ & \multirow[t]{5}{*}{$\begin{array}{l}\text { No difference between } \\
\text { the two groups }\end{array}$} & Study Quality - 8/10 \\
\hline & & \multirow{4}{*}{$\begin{array}{l}\text { Nurse led telephone } \\
\text { follow up plus educational } \\
\text { group programme (EGP) }\end{array}$} & & & \multirow{4}{*}{$\begin{array}{l}\text { Secondary measures } \\
\text { included role and emotional } \\
\text { functioning and feelings of } \\
\text { control and anxiety }\end{array}$} & & External validity $-2 / 3$ \\
\hline & & & & & & & $\begin{array}{c}\text { Internal Validity } \\
\text { (bias) }-5 / 7\end{array}$ \\
\hline & & & & & & & $\begin{array}{c}\text { Internal Validity } \\
\text { (selection bias) }-6 / 6\end{array}$ \\
\hline & & & & & & & $\begin{array}{l}\text { Power - 1/1 } \\
\text { (Total - 22/27) }\end{array}$ \\
\hline \multirow{2}{*}{$\begin{array}{l}\text { Kimman et al., } \\
2011 \text { Maastricht, } \\
\text { Netherlands [27] }\end{array}$} & \multirow{2}{*}{$\begin{array}{l}299 \text { women } \\
\text { with breast } \\
\text { cancer }\end{array}$} & \multirow{2}{*}{$\begin{array}{l}\text { Nurse led telephone } \\
\text { follow up or Nurse led } \\
\text { telephone follow up plus } \\
\text { educational group } \\
\text { programme (EGP) }\end{array}$} & \multirow{2}{*}{$\begin{array}{l}\text { Hospital follow up or } \\
\text { hospital follow up } \\
\text { plus EGP }\end{array}$} & \multirow[t]{2}{*}{18 months } & $\begin{array}{l}\text { Quality adjusted life } \\
\text { gain (QALYs) }\end{array}$ & \multirow{2}{*}{$\begin{array}{l}\text { Hospital follow-up plus } \\
\text { EGP resulted in the highest } \\
\text { QALYs but has the highest } \\
\text { costs. Next best in terms of } \\
\text { costs and QALYs was nurse } \\
\text { led telephone follow up } \\
\text { plus EGP }\end{array}$} & \multirow[t]{2}{*}{$\begin{array}{l}\text { No score as cost } \\
\text { analysis }\end{array}$} \\
\hline & & & & & $\begin{array}{l}\text { Incremental cost-effectiveness } \\
\text { ratios (ICERs) }\end{array}$ & & \\
\hline \multirow{4}{*}{$\begin{array}{l}\text { Kimman et al., } \\
2010 \text { Maastricht, } \\
\text { Netherlands [13] }\end{array}$} & \multirow{4}{*}{$\begin{array}{l}299 \text { women } \\
\text { with breast } \\
\text { cancer }\end{array}$} & $\begin{array}{l}\text { Nurse led telephone } \\
\text { follow up or }\end{array}$ & \multirow{4}{*}{$\begin{array}{l}\text { Hospital follow up or } \\
\text { hospital follow up } \\
\text { plus EGP }\end{array}$} & \multirow[t]{4}{*}{12 months } & \multirow[t]{4}{*}{ Patient satisfaction } & \multirow{4}{*}{$\begin{array}{l}\text { Increased patient } \\
\text { satisfaction with access } \\
\text { to care in telephone } \\
\text { follow-up group. No } \\
\text { significant influence on } \\
\text { general patient satisfaction, } \\
\text { technical competence or } \\
\text { inter-personal aspects }\end{array}$} & Study Quality - 9/10 \\
\hline & & \multirow{3}{*}{$\begin{array}{l}\text { Nurse led telephone } \\
\text { follow up plus educational } \\
\text { group programme (EGP) }\end{array}$} & & & & & External validity $-2 / 3$ \\
\hline & & & & & & & $\begin{array}{l}\text { Internal Validity } \\
\text { (bias) }-5 / 7\end{array}$ \\
\hline & & & & & & & $\begin{array}{c}\text { Internal Validity } \\
\text { (selection bias) }-5 / 6\end{array}$ \\
\hline
\end{tabular}


Table 1 Details of included studies including critical appraisal scores (Continued)

\begin{tabular}{|c|c|c|c|c|c|c|c|}
\hline & & & & & & & $\begin{array}{l}\text { Power - 1/1 } \\
\text { (Total - 22/27) }\end{array}$ \\
\hline \multirow{5}{*}{$\begin{array}{l}\text { Kroenke et al., } \\
2010 \text { Indiana, } \\
\text { USA [26] }\end{array}$} & \multirow{5}{*}{$\begin{array}{l}405 \text { cancer } \\
\text { patients }\end{array}$} & \multirow{5}{*}{$\begin{array}{l}\text { Centralized telecare } \\
\text { management by a } \\
\text { nurse-physican specialist } \\
\text { team coupled with } \\
\text { home-based symptom } \\
\text { monitoring by interactive } \\
\text { voice recording or internet }\end{array}$} & \multirow[t]{5}{*}{ Usual care } & \multirow[t]{5}{*}{12 months } & \multirow[t]{5}{*}{ Depression Pain } & \multirow{5}{*}{$\begin{array}{l}\text { Improvements in pain } \\
\text { and depression for the } \\
\text { intervention group }\end{array}$} & Study Quality - 8/10 \\
\hline & & & & & & & External validity $-2 / 3$ \\
\hline & & & & & & & $\begin{array}{l}\text { Internal Validity } \\
\text { (bias) 6/7- }\end{array}$ \\
\hline & & & & & & & $\begin{array}{l}\text { Internal Validity } \\
\text { (selection bias) }-6 / 6\end{array}$ \\
\hline & & & & & & & $\begin{array}{c}\text { Power }-1 / 1 \text { (Total - } \\
23 / 27 \text { ) }\end{array}$ \\
\hline \multirow[t]{5}{*}{$\begin{array}{l}\text { Marcus et al., } 2009 \\
\text { Colorado, USA [18] }\end{array}$} & \multirow[t]{5}{*}{$\begin{array}{l}304 \text { breast } \\
\text { cancer patients }\end{array}$} & \multirow[t]{5}{*}{$\begin{array}{l}16 \text { session telephone } \\
\text { counselling post treatment }\end{array}$} & \multirow{5}{*}{$\begin{array}{l}\text { Resource directory for } \\
\text { breast cancer was } \\
\text { given to each patient }\end{array}$} & \multirow[t]{5}{*}{18 months } & Distress & $\begin{array}{l}\text { No difference for distress } \\
\text { and depression }\end{array}$ & Study Quality - 8/10 \\
\hline & & & & & Depression & $\begin{array}{l}\text { Need for clinical referral - } \\
\text { depression and distress } \\
\text { reduced by } 50 \% \text { in the } \\
\text { intervention group for } \\
\text { dichotomized end points }\end{array}$ & External validity $-2 / 3$ \\
\hline & & & & & Sexual dysfunction & \multirow{3}{*}{$\begin{array}{l}\text { Effects found for personal } \\
\text { growth and sexual } \\
\text { dysfunction in the } \\
\text { intervention group }\end{array}$} & $\begin{array}{l}\text { Internal Validity } \\
\text { (bias) }-5 / 7\end{array}$ \\
\hline & & & & & \multirow[t]{2}{*}{ Personal growth } & & $\begin{array}{l}\text { Internal Validity - } 5 / 6 \\
\text { (selection bias) }\end{array}$ \\
\hline & & & & & & & $\begin{array}{l}\text { Power - 0/1 } \\
\text { (Total - 20/27) }\end{array}$ \\
\hline \multirow[t]{6}{*}{$\begin{array}{l}\text { Matthew et al., } 2007 \\
\text { Toronto, Canada [20] }\end{array}$} & \multirow[t]{6}{*}{$\begin{array}{l}152 \text { prostate } \\
\text { cancer patients }\end{array}$} & $\begin{array}{l}\text { PDA survey followed } \\
\text { by paper }\end{array}$ & \multirow[t]{6}{*}{$\begin{array}{l}\text { Paper followed } \\
\text { by PDA survey }\end{array}$} & \multirow[t]{6}{*}{30 mins } & \multirow{6}{*}{$\begin{array}{l}\text { Survey was monitoring } \\
\text { health-related quality of life } \\
\text { but outcomes looked at } \\
\text { assessment of data quality } \\
\text { and feasibility }\end{array}$} & Internal consistency similar & Study Quality - 8/10 \\
\hline & & $\begin{array}{l}\text { PDA followed by PDA } \\
\text { survey. (3 groups) }\end{array}$ & & & & $\begin{array}{l}\text { Test re-test reliability } \\
\text { confirmed }\end{array}$ & External validity - $3 / 3$ \\
\hline & & & & & & $\begin{array}{l}\text { Data from two modalities } \\
\text { strongly correlated. }\end{array}$ & $\begin{array}{l}\text { Internal Validity } \\
\text { (bias) }-5 / 7\end{array}$ \\
\hline & & & & & & $\begin{array}{l}\text { Fewer missed items for } \\
\text { the PDA }\end{array}$ & $\begin{array}{l}\text { Internal Validity } \\
\text { (selection bias) }-5 / 6\end{array}$ \\
\hline & & & & & & $\begin{array}{l}\text { More preferred using the } \\
\text { PDA or had no preference. } \\
\text { PDA found easy to use }\end{array}$ & $\begin{array}{l}\text { Power - 0/1 } \\
\text { (Total - 21/27) }\end{array}$ \\
\hline & & & & & & $\begin{array}{l}\text { Age did not correlate } \\
\text { with difficulty using PDA }\end{array}$ & \\
\hline
\end{tabular}


Table 1 Details of included studies including critical appraisal scores (Continued)

\begin{tabular}{|c|c|c|c|c|c|c|c|}
\hline \multirow[t]{4}{*}{$\begin{array}{l}\text { Sandgren et al., } \\
2003 \text { North Dakota, } \\
\text { USA [19] }\end{array}$} & \multirow[t]{4}{*}{$\begin{array}{l}222 \text { women } \\
\text { with breast } \\
\text { cancer }\end{array}$} & \multirow[t]{4}{*}{$\begin{array}{l}6 \times 30 \text { min telephone } \\
\text { therapy sessions that } \\
\text { involved either cancer } \\
\text { education or emotional } \\
\text { expressions }\end{array}$} & \multirow[t]{4}{*}{ Standard care } & \multirow[t]{4}{*}{5 months } & $\begin{array}{l}\text { Perceived control } \\
\text { Mood }\end{array}$ & $\begin{array}{l}\text { Cancer education group } \\
\text { reported greater perceived } \\
\text { control compared to } \\
\text { standard care }\end{array}$ & Study Quality - 7/10 \\
\hline & & & & & \multirow[t]{3}{*}{ Quality of life } & \multirow[t]{3}{*}{$\begin{array}{l}\text { No difference for mood } \\
\text { or quality of life }\end{array}$} & $\begin{array}{l}\text { External validity }-2 / 3 \\
\text { Internal Validity } \\
\text { (bias) }-5 / 7\end{array}$ \\
\hline & & & & & & & $\begin{array}{l}\text { Internal Validity } \\
\text { (selection bias) }-6 / 6\end{array}$ \\
\hline & & & & & & & $\begin{array}{l}\text { Power - 1/1 } \\
\text { (Total - 21/27) }\end{array}$ \\
\hline \multirow{5}{*}{$\begin{array}{l}\text { Sikorski et al., } 2009 \\
\text { Michigan, USA [22] }\end{array}$} & \multirow{5}{*}{$\begin{array}{l}486 \text { cancer } \\
\text { patients }\end{array}$} & \multirow{5}{*}{$\begin{array}{l}\text { Automated voice response } \\
\text { symptom reporting }\end{array}$} & \multirow{5}{*}{$\begin{array}{l}\text { Nurse assisted symptom } \\
\text { management via the } \\
\text { telephone }\end{array}$} & \multirow{5}{*}{$\begin{array}{l}6 \text { telephone } \\
\text { contacts over } \\
8 \text { weeks }\end{array}$} & \multirow{5}{*}{$\begin{array}{l}\text { Severity of cancer symptom } \\
\text { at intake interview and at } \\
\text { first intervention contact }\end{array}$} & \multirow{5}{*}{$\begin{array}{l}\text { Patient in the AVR group } \\
\text { reported more severe } \\
\text { symptoms. There was a } \\
\text { variation with age with } \\
\text { older patients reporting } \\
\text { more severity of symptoms } \\
\text { to the nurse }\end{array}$} & Study Quality - 9/10 \\
\hline & & & & & & & External validity - $2 / 3$ \\
\hline & & & & & & & $\begin{array}{l}\text { Internal Validity } \\
\text { (bias) }-5 / 7\end{array}$ \\
\hline & & & & & & & $\begin{array}{l}\text { Internal Validity } \\
\text { (selection bias) }-6 / 6\end{array}$ \\
\hline & & & & & & & $\begin{array}{l}\text { Power - 0/1 } \\
\text { (Total - 22/27) }\end{array}$ \\
\hline \multirow{5}{*}{$\begin{array}{l}\text { Sikorskii et al., } 2007 \\
\text { Michigan, USA [23] }\end{array}$} & \multirow{5}{*}{$\begin{array}{l}435 \text { cancer } \\
\text { patients }\end{array}$} & \multirow{5}{*}{$\begin{array}{l}\text { Automated telephone } \\
\text { symptom management }\end{array}$} & \multirow{5}{*}{$\begin{array}{l}\text { Nurse-assisted symptom } \\
\text { management }\end{array}$} & \multirow[t]{5}{*}{10 weeks } & \multirow{5}{*}{$\begin{array}{c}\text { Severity of cancer symptoms, } \\
\text { demographic data and } \\
\text { co-morbidities }\end{array}$} & \multirow{5}{*}{$\begin{array}{l}\text { Reduction in symptom } \\
\text { severity in both groups. Lung } \\
\text { cancer patients with greater } \\
\text { symptom severity withdrew } \\
\text { from the ATSM group }\end{array}$} & Study Quality - 8/10 \\
\hline & & & & & & & External validity $-2 / 3$ \\
\hline & & & & & & & $\begin{array}{l}\text { Internal Validity } \\
\text { (bias) }-5 / 7\end{array}$ \\
\hline & & & & & & & $\begin{array}{c}\text { Internal Validity } \\
\text { (selection bias) }-6 / 6\end{array}$ \\
\hline & & & & & & & $\begin{array}{l}\text { Power - 1/1 } \\
\text { (Total - 22/27) }\end{array}$ \\
\hline \multirow{5}{*}{$\begin{array}{c}\text { Yun et al. } 2012 \text { Seoul, } \\
\text { Korea [24] }\end{array}$} & \multirow{5}{*}{$\begin{array}{l}273 \text { cancer } \\
\text { patients }\end{array}$} & \multirow{5}{*}{$\begin{array}{l}\text { Internet based, individually } \\
\text { tailored cancer related } \\
\text { fatigue education program }\end{array}$} & \multirow[t]{5}{*}{ Usual care } & \multirow[t]{5}{*}{12 weeks } & Level of fatigue & \multirow{5}{*}{$\begin{array}{l}\text { Education group reported } \\
\text { a reduction in fatigue, } \\
\text { decrease in HADS anxiety } \\
\text { score, increase in global } \\
\text { QoL score and emotional, } \\
\text { cognitive and social } \\
\text { functioning of EORTIC } \\
\text { QLQ-C30 }\end{array}$} & Study Quality - 8/10 \\
\hline & & & & & Quality of Life, Anxiety & & External validity - 1/3 \\
\hline & & & & & and depression & & $\begin{array}{l}\text { Internal Validity } \\
\text { (bias) }-4 / 7\end{array}$ \\
\hline & & & & & & & $\begin{array}{c}\text { Internal Validity } \\
\text { (selection bias) }-6 / 6\end{array}$ \\
\hline & & & & & & & $\begin{array}{l}\text { Power - 1/1 } \\
\text { (Total - 20/27) }\end{array}$ \\
\hline
\end{tabular}




\begin{tabular}{|c|c|c|c|c|}
\hline Study & Intervention & Primary and secondary outcomes & Measures used & Results including statistical values \\
\hline \multirow[t]{5}{*}{ Beaver et al. (2009) $[11,25]$} & \multirow{5}{*}{$\begin{array}{l}\text { Telephone follow up by } \\
\text { specialist nurses }\end{array}$} & Psychological Morbidity & State-trait anxietntdy inventory & No difference for psychological morbidity \\
\hline & & Participant's need for information & \multirow[t]{4}{*}{ General Health Questionnaire } & $\begin{array}{l}\text { Patients in telephone group more satisfied } \\
\text { (intention to treat } p<0.001 \text { ) }\end{array}$ \\
\hline & & Participants' satisfaction & & No difference for information needs \\
\hline & & Clinical investigations ordered & & No difference for clinical investigations \\
\hline & & Time to detection of recurrent disease & & $\begin{array}{l}\text { Recurrences :- no differences between the two } \\
\text { groups } p=0.295 \% \mathrm{Cl}(-3.33-2.07) \text { - equivalence } \\
\text { demonstrated. 28) }\end{array}$ \\
\hline \multirow[t]{3}{*}{ Davison et al. (2002) [15] } & \multirow{3}{*}{$\begin{array}{l}\text { Computer programme } \\
\text { providing information } \\
\text { and assisting decision } \\
\text { making }\end{array}$} & $\begin{array}{l}\text { "Extent to which women achieved their } \\
\text { preferred decisional roles" }\end{array}$ & Control Preferences Scale (CPS) & $\begin{array}{l}\text { Intervention group was more passive in decision } \\
\text { making than planned. }(p<0.0001)\end{array}$ \\
\hline & & \multirow[t]{2}{*}{ Patient satisfaction } & \multirow[t]{2}{*}{ Patient Satisfaction Questionnaire (PSQ) } & $\begin{array}{l}\text { More women over } 50 \text { opted to play a more } \\
\text { passive role. }(p=>0.002)\end{array}$ \\
\hline & & & & $\begin{array}{l}\text { No difference found for the two groups for } \\
\text { patient satisfaction. Both groups reported } \\
\text { high levels }\end{array}$ \\
\hline \multirow[t]{5}{*}{ Harrison et al. (2011) [21] } & \multirow[t]{5}{*}{$\begin{array}{l}5 \text { telephone calls from a } \\
\text { specialist colorectal nurse } \\
\text { in } 6 \text { months after discharge }\end{array}$} & Unmet supportive care needs & $\begin{array}{l}\text { SCNS-SF34 and FACT-C used for } \\
\text { unmet supportive care needs } \\
\text { and quality of life. CaSUN was } \\
\text { used to measure these two } \\
\text { outcomes at } 6 \text { months }\end{array}$ & $\begin{array}{l}\text { No difference was found for unmet supportive } \\
\text { care needs at } 6 \text { months }\end{array}$ \\
\hline & & Secondary outcomes: & \multirow{4}{*}{$\begin{array}{l}\text { Patient asked to remember } \\
\text { health service use in a } \\
\text { telephone interview }\end{array}$} & $\begin{array}{l}\text { "Observed effect size for supportive care needs } \\
\text { was } 0.25 \text { " }\end{array}$ \\
\hline & & Health service utilization & & Study was aiming for effect size of 0.75 . \\
\hline & & \multirow[t]{2}{*}{ Quality of life } & & $\begin{array}{l}\text { Quality of life had improved by "twice as much" } \\
\text { in the intervention group at six months. (size of } \\
\text { difference }=5.7 \text { ) }\end{array}$ \\
\hline & & & & $\begin{array}{l}\text { At } 6 \text { months in the intervention group; fewer } \\
\text { "presentations to emergency departments } \\
(p=0.23) \text { and readmissions to hospital ( } p=0.37) \text { " } \\
\text { compared with the control group. Intervention } \\
\text { group patients had more contact with } \\
\text { "hospital-based, specialist based and community } \\
\text { services". Differences for health service utilization } \\
\text { were not statistically significant }\end{array}$ \\
\hline \multirow[t]{3}{*}{ Hegel et al. (2010) [16] } & \multirow{3}{*}{$\begin{array}{l}6 \text { weekly session of } \\
\text { telephone delivered } \\
\text { problem solving } \\
\text { occupational therapy }\end{array}$} & $\begin{array}{l}\text { Feasibility of conducting a RCT including } \\
\text { patient satisfaction }\end{array}$ & $\begin{array}{l}\text { Study recruitment and retention } \\
\text { data was gathered }\end{array}$ & $" 67 \%$ recruitment rate $(31 / 46) "$ \\
\hline & & Secondary outcomes: & \multirow{2}{*}{$\begin{array}{l}\text { At } 12 \text { weeks participants } \\
\text { completed a satisfaction survey }\end{array}$} & " $81 \%$ retention rate" \\
\hline & & Functional quality of life & & $\begin{array}{l}\text { "92\% of those receiving the intervention were } \\
\text { "highly satisfied" }\end{array}$ \\
\hline
\end{tabular}


Kimman et al., 2011 [17,27]

Nurse led telephone follow up or

Nurse led telephone follow up plus educational group programme (EGP)

Kroenke et al. (2010) [26]

Centralized telecare management by a nursephysican specialist team coupled with home-based symptom monitoring by interactive voice recording or internet
Electronic symptom questionnaire completed by patients in control and intervention group before the start of chemotherapy and prior to cycles 2, 3, 4 and 5 mucositis, hand-foot syndrome and diarrhoea"

Health-related quality of life ( $\mathrm{HROOL}$ )

Secondary outcomes:

Role and emotional functioning

Feelings of control and anxiety

Depression and pain

Secondary outcomes:

Health-related quality of life

Disability

Cointerventions

Self reported health care use

HROOL: EORTC OLQ-C3O

Role and emotional functioning: EORTC OLQ-C30 subscales (STAl)

Perceived feelings of contro:

Mastery scale

Measured at baseline and at 1, 3, 6 and 12 months. Depression measured using the "20-item Hopkins Symptom Checklist (HSCL-20) and pain (BPI) severity

"Quality of life - single item 0-10" Anxiety - "7-item Generalised Anxiety Disorder scale" somatic symptom scale"

Fatigue- "SF 36 vitality scale"
92\% reported it to be "helpful/very helpful"

$97 \%$ if planned sessions of the intervention were completed

Effect sizes were calculated for secondary outcomes but "study was not powered to detect treatment effects". Main outcome was feasibility for study to be repeated as larger scale RCT. No Cls quoted

In the control group more report of fatigue $(\mathrm{Cl}=1.04-5.05, \mathrm{P}=0.040)$ and lower reporting of hand-foot syndrome $(\mathrm{Cl}$ 0.17-0.92 $\mathrm{P}=0.031)$ Severity and distress of symptoms were no different between the two groups except for hand-foot syndrome in the intervention group. (Severity $\mathrm{Cl}-0.52$ to $-0.02 \mathrm{P}=0.033$, Distress $\mathrm{Cl}-0.33$ to $-0.02, \mathrm{P}=0.028)$. Other differences were not statistically significant

No difference between the two groups for HROOL (P value $=0.42$

Confidence interval of 95\% for the "estimated difference between mean HRQoL scores at 12 months after treatment" $=-1.93-4.64$ )

Anxiety: State Trait Anxiety Inventon

No differences between groups for all other secondary outcomes (all $p$ values $>0.05$ ) Health related quality of life: SF-12

"Physical symptom burden - 22-item

Disability - "3- item Sheehan Disability Scale"

"Self-report health care use: treatment survey" 
Table 2 Details of intervention, outcomes, measures used and results (Continued)

Marcus et al. (2009) [18] 16 session telephone Cancer specific Distress counselling post treatment

Depression

Sexual dysfunction

Personal Growth

Matthew et al. (2007) [20]

PDA survey followed by paper

Data quality

PDA followed by PDA

survey. (3 groups)

Feasibility

$6 \times 30$ min telephone

therapy sessions that

involved either cancer

education or emotional

expressions

Mood
Cancer specific Distress - Impact of Event Scale (IES).

Depression - Centre for Epidermiologic Studies Depression Scale (CES-D)

Sexual Dysfunction - 25 items (designed for study)

Personal growth - 5 items (designed for study)

International Prostate Symptom Score (IPSS)

Patient Orientated Prostate cancer Utility Survey (PORPUS) Internationa Index of Erectile Function-5 (IIEF-5) either in paper or PDA forms

Quality of Life - Functional Assessment of Cancer Therapy-Breast Instrument (FACT-B)

Mood - Profile of Mood States
No differences found for depression and distress unless end points were "dichotomized at cutpoints suggestive of the need for clinical referral". A 50\% reduction in depression and distress was demonstrated in the intervention group compared to the control. $(p=0.07)$

"Significant effects" shown in sexual dysfunction and personal growth for the intervention group

When endpoints dichotomized- no change in the control group (depression: $p=0.41$ distress $=0.86$ ). Intervention group (depression $p=0.0007$, distress $p=0.0007$ )

Group differences at 18 months were significant Depression: $p=0.06$ and Distress: $p=0.07$ "with effect sizes of 0.23 and 0.24

Sexual dysfunction: at 18 months, "significant improvement intervention group" $p=0.04$, effect size $=0.23$

Personal growth- both groups improved but more in the intervention group. (At 18 months $p=0.03$, effect size $=0.22$ )

Internal consistency found to be high

Test re-test reliability high. $(p<0.01)$

Scores across modalities were correlated demonstrating "concurrent validity $(p<0.01)$ "

No differences in levels of participation

Preference was highest for the PDA version of the questionnaire. (58.6\%)

Age did not have an impact on preference $(p=0.12)$

Age did not have an impact of difficulty using PDA. $(p=0.08)$

Confidence intervals quoted in the paper for each of the data items within the questionnaire

Cancer Education group - greater perceived control $(p<0.01)$

No difference for mood ( $p>0.12$ ) or quality of life $(p>0.12)$ found 
Table 2 Details of intervention, outcomes, measures used and results (Continued)

\begin{tabular}{|c|c|c|c|c|}
\hline & & Perceived control & $\begin{array}{l}\text { Perceived control - Perceived } \\
\text { Stress Scale }\end{array}$ & $\begin{array}{l}\text { No Cls quoted- only standard deviations, means } \\
\text { and } p \text { values }\end{array}$ \\
\hline \multirow[t]{2}{*}{ Sikorskii et al. (2007) [22] } & \multirow[t]{2}{*}{$\begin{array}{l}\text { Automated telephone } \\
\text { symptom management }\end{array}$} & \multirow[t]{2}{*}{ Severity of symptoms } & \multirow{2}{*}{$\begin{array}{l}17 \text { symptoms scored for severity - } \\
\text { designed for the study. Analysed } \\
\text { using a RASCH model }\end{array}$} & $\begin{array}{l}\text { Both groups had a reduction in symptom } \\
\text { severity. No difference found between } 2 \text { groups }\end{array}$ \\
\hline & & & & $\begin{array}{l}\text { Effect sizes were almost the same for NASM } \\
(0.56) \text { and ATSM }(0.59)\end{array}$ \\
\hline \multirow[t]{2}{*}{ Sikorskii et al. (2009) [23] } & \multirow[t]{2}{*}{$\begin{array}{l}\text { Automated voice response } \\
\text { symptom reporting }\end{array}$} & \multirow[t]{2}{*}{$\begin{array}{l}\text { Severity of symptoms - difference } \\
\text { depending on mode of assessment }\end{array}$} & \multirow[t]{2}{*}{$\begin{array}{l}14 \text { cancer related symptoms - } \\
\text { scored for severity. Designed } \\
\text { for the study }\end{array}$} & $\begin{array}{l}\text { AVR group reported more severe symptoms } \\
\text { of "nausea, vomiting, diarrhoea, poor appetite, } \\
\text { pain and alopecia ( } p \text { values less than } 0.05) \text { " }\end{array}$ \\
\hline & & & & $\begin{array}{l}\text { Varied with age with older patients reporting } \\
\text { higher severity in the nurse led group (effect } \\
\text { sizes greater or equal to } 0.35^{\prime \prime} \text { ) }\end{array}$ \\
\hline \multirow[t]{4}{*}{ Yun et al. 2012 Seoul, Korea [24] } & \multirow[t]{4}{*}{$\begin{array}{l}\text { Internet based, individually } \\
\text { tailored cancer related fatigue } \\
\text { education program }\end{array}$} & Level of fatigue & Brief Fatigue Inventory (BFI) & $\begin{array}{l}\text { Intervention group reported an improvement } \\
\text { in fatigue with a significantly greater decrease } \\
\text { in BFI global score }(-0.66 \text { points, } 95 \% \mathrm{Cl}-1.04 \\
\text { to }-0.27), \text { FSS total score }(-0.49 ; 95 \% \mathrm{Cl}, 0.78 \\
\text { to }-0.21) \text { and HADS score }\end{array}$ \\
\hline & & Anxiety and depression & Fatigue Severity Scale (FSS) & \multirow{3}{*}{$\begin{array}{l}\text { Participants with moderate or greater fatigue } \\
\text { reported a significantly greater decrease in } \\
\text { HADS Anxiety score (-0.90; } 95 \% \mathrm{Cl},-1.51 \\
\text { to }-0.29) \text { as well as global quality of life }(5.22 \text {; } \\
95 \% \text { Cl, } 0.93 \text { to } 9.50) \text { and several functioning } \\
\text { scores of the EORTC QLQ_C } 30\end{array}$} \\
\hline & & \multirow[t]{2}{*}{ Global quality of life } & $\begin{array}{l}\text { Hospital Anxiety and Depression } \\
\text { Scale (HADS) }\end{array}$ & \\
\hline & & & $\begin{array}{l}\text { European Organisation for } \\
\text { Research and Treatment of } \\
\text { Cancer Quality of Life } \\
\text { Questionnaire C30 } \\
\text { (EORTC QLQ_C30) }\end{array}$ & \\
\hline
\end{tabular}


to face follow-up meeting, the aim being to enable them to participate more fully in their subsequent follow-up consultation [15]. One study sought to combine technologies in a centralised telephone management system operated by a specialist nurse [26]. Patients' symptoms were monitored in their own home using an internet based application and/or a voice activated telephone helpline with nurses telephoning patients back if symptoms indicated this was required. The final study developed Health Navigation, an Internet-based individually tailored education programme for patients suffering from cancer related fatigue. Participants had access to the Health Navigation website and were guided through a twelve week programme including sessions on energy conservation, physical activity, nutrition, sleep hygiene, pain control and distress management [24].

\section{Patient acceptability/satisfaction}

Data on patient satisfaction or acceptability was explicitly reported in five of the trials $[11,13,15,16,25]$. Beaver et al. reported that most women with breast cancer had equivalent satisfaction with elements of cancer followup received via technological means compared to usual care $[11,25]$. Kimman et al. found that nurse-led telephone follow-up after curative treatment for breast cancer resulted in high satisfaction scores with the added potential to reduce clinic visits [13]. In the study of symptom monitoring using paper or PDA surveys in men with prostate cancer, most preferred using the PDA and found it easy to use, and older age did not appear to be associated with reports of difficulty in using the PDA [15]. A smaller feasibility trial, reported that $92 \%$ of US women with breast cancer who received 6 weeks of telephone delivered problem solving occupational therapy were highly satisfied with the intervention [16]. There was no evidence from the other studies that technological interventions led to reduced patient satisfaction with follow-up care.

\section{Clinical safety}

Only one study reported on clinical safety $[11,25]$. It was conducted amongst 374 women with breast cancer and reported on time to detection of recurrence, with no significant differences observed between intervention and control groups.

\section{Health-related quality of life}

In a Scottish study of remote monitoring of symptoms in patients undergoing chemotherapy intervention group patients were less fatigued and reported more hand and foot syndrome [12]. Similarly, in a US trial of chemotherapy related symptom monitoring patients receiving symptom monitoring via an automated telephone symptom monitoring system reported more severe symptoms than those receiving nurse assisted symptom management [22,23].
After 10 weeks symptom severity, compared to baseline, had reduced in both groups [23]. An Australian study of patients with colorectal cancer receiving telephone followup from a specialist nurse, quality of life scores were higher in the intervention group at six months [21]. Intervention participants in the Korean fatigue study [24] reported a decrease in 12 week scores compared to baseline in both the Brief Fatigues Inventory and Fatigue Severity Scale as well as a reduction in anxiety and several functioning scores of the EORTC-C30. However a study of 299 breast cancer patients in the Netherlands reported no significant difference in the health-related quality of life between the intervention group receiving telephone follow up and the control group receiving hospital follow up [17]. Taking these six trials together there was no evidence of significantly increased psychological distress or reduced quality of life in any of the intervention groups.

\section{Health economic outcomes}

Only two studies included any assessment of health economic outcomes with only one study reporting specifically on cost [25]. Beaver et al. conducted a cost minimisation analysis of specialist nurse-led follow-up [25]. This concluded that telephone follow-up was more costly for health services, with patients receiving telephone follow-up having approximately $20 \%$ more consultations than those receiving hospital follow-up, in addition telephone consultations were longer than clinic consultations and requests for mammography or additional referrals were greater in this group, however telephone follow-up was less costly for patients [25]. It should be noted that many of the health service costs related to training to deliver the intervention, costs which would subsequently decline [25]. On the other hand, Dutch investigators, Kimman et al., [27] concluded that standard hospital follow-up plus an educational group programme resulted in greater gain in QALYs compared to telephone follow-up, but at considerable cost possibly due to the high level of contacts made by the telephone follow-up group with specialised healthcare professionals and their costs of lost production [27]. In a subgroup analysis included within this study hospital follow-up and an educational group programme was found to be maximally cost effective in anxious patients. Age, level of education, and chemotherapy did not influence cost-effectiveness [27]. Beaver et al. did not conduct a sub-group analysis so comparisons could not be made at this level [25].

In the other studies, whilst not formally reporting any health economic evaluation, there was no evidence of increased health care utilisation amongst recipients of the intervention in fact one noted a significant reduction in subsequent referrals for depression and distress amongst 304 US breast cancer patients that had received telephone follow-up [18]. 


\section{Critical appraisal of studies}

A standard checklist was used to critically appraise the included studies using standard criteria [14]. Scores are shown in Table 1 and ranged from 17 to 23. One study attracted a quality score of $6 / 10$, mainly due to poor expression of the study aims and description of the participants [15]. Thus, these studies were generally of good quality and reported to a high standard. In all cases the researchers had addressed issues of internal validity and recognised the issues created by not being able, in most, cases to blind participants to the intervention. Most studies failed to employ blinded data collection. In all studies there was good recognition of, and controlling of, potential confounding leading to high scores in this domain. Most of the studies were, however, underpowered with respect to clinical outcomes. The main issue of quality with the study was a failure to demonstrate external validity. Only four of the included studies included information on non-respondents, generally reporting that they were older and of lower educational status $[11,17,18,20]$. This underscores the fact that technology trials as currently reported exhibit considerable recruitment bias. This was further illustrated by some of the studies not reporting on non-respondents acknowledging that this was a limitation and highlighting the fact that they had, for example, recruited only urban or white participants $[15,18,28]$.

\section{Discussion}

\section{Summary of evidence}

The limited evidence available suggests that using technology for cancer follow-up is acceptable to patients and clinically safe. However, there is currently insufficient evidence to definitively state whether or not remote cancer follow-up using technology is likely to be cost effective, since most existing randomised trial evidence relates to alternative models of follow-up using standard telephone calls only. The results are important as a prompt and guide to future research since, in the face an ageing population and increasing number of cancer survivors, the need for safe, acceptable and economically viable alternatives to current resource intensive cancer follow-up care delivery models is urgent. Existing evidence suggests that scheduled cancer follow-up can employ elements of technology without reducing patient satisfaction, compromising safety, impairing quality of life or increasing psychological distress.

\section{Strengths and limitations}

As far as we aware this is the first systematic review to focus on the use of technology to deliver cancer followup. The methods of the review were thorough and robust and the authors have confidence that the search strategy was suitably inclusive to identify most relevant studies.
We chose to include only randomised controlled trials in the review and did not include other studies types, such as interrupted time series studies. This was principally due to our concern that trials of new technologies are particularly prone to selection bias (i.e. the recruitment of enthusiasts). Whilst the randomised trial is not a panacea to this problem in technological research we believe that by restricting our review to a method which explicitly addresses this issue was the best approach. A further issue is that the CONSORT statement which promotes quality in the reporting has been widely accepted and implemented. Furthermore randomised trials, in general, include harder outcome. We believe our results have borne out our approach.

The review was limited to studies written in English. Thus it is possible that some studies conducted in other parts of the world were missed. This is particularly so when it is considered that many non-English speaking countries, for example, Germany are key-players in technological innovation. We do think, however, that since English is practically the "lingua franca" of medical research it is unlikely that we have missed any major randomised trial by employing this limitation. It does seem most likely that these would have been published in a journal employing the English language for which the search strategy was exhaustive. Further support for this view can be obtained from the fact that both trials currently registered on the ISRCTN register of using technology in cancer follow-up are in non-English speaking countries but fully described and registered in English.

The majority of existing studies were conducted in the developed world in women with breast cancer, a group which will potentially represent the more enabled and affluent end of the cancer survivorship spectrum. This limits the extension of the current findings to other settings, cancers and patient groups, emphasising the need for further research. It could also be that there are patient groups in whom it would be inappropriate to attempt to utilise technology in their follow-up, an issue that the current evidence goes no way toward identifying or addressing. Furthermore, there was insufficiently consistent reporting within the studies of inclusion criteria with respect to time since completion of treatment or stage at diagnosis to draw meaningful conclusions with respect to these parameters. A further limitation has occurred in the synthesis and interpretation of the evidence. There were surprisingly few randomised studies identified and the majority used telephone follow-up only, with only a few adopting more recent technological innovations such as PDAs. Thus, given the increasing potential of modern digital technologies the included interventions were relatively simple and low-tech. Furthermore, the way in which technology could enable patients to send information to healthcare professionals has not yet been meaningfully 
explored. Although the interventions addressed several aspects of follow-up care (recurrence monitoring, monitoring treatment effect, information provision) none were comprehensive, and most used technology to deliver one aspect of follow-up care as an adjunct to more traditional follow-up models. Because of this direct comparison of interventions was extremely difficult given the different technologies, interventions and outcomes reported. Nevertheless, we believe the current review provides good evidence of the potential of technology to deliver safe effective cancer follow-up in the future. The review also highlights the need for future trials to seek to provide definitive information on patient acceptability, clinical safety and cost effectiveness.

\section{Context with other literature}

There is a growing recognition that cancer follow-up services are increasingly stretched by a growing population of cancer survivors $[1,3,6]$. This has prompted researchers to develop alternative models of care which have utilised different healthcare professionals in care delivery and/or shifted the focus of care from hospital to the community $[4,29,30]$. Despite, this, these models will probably continue to consume a large and growing amount of healthcare resource and still inconvenience certain patient groups. The randomised studies included within this review are striking in the respect that they demonstrate that few mature complex interventions utilising technology, other than standard telephone calls, have been developed to a standard sufficient for subjection to a randomised controlled trial. The need to do this is, perhaps, one of the key implications of our work. There is an emerging vision that modern technologies, evidenced by policy initiative worldwide, could potentially enhance current models or offer other alternatives to the future delivery of high quality cancer care [31-38]. There is considerable evidence from other disease areas of using modern technologies to good effect [39]. For example, text messaging has been found to increase self-efficacy and adherence to medication in young people with diabetes and has been proven to be an effective aid to smoking cessation [40,41]. Online systems which enable patients to access their own health records have enjoyed some success as a means to educate patients and assist in their self-management, for example, in the USA, Kaiser Permanente's My Health Manager has demonstrated significant reductions in primary care contacts [42]. The CHAMPION project has enabled disabled adults to improve communication with healthcare professionals by inputting information on their own care needs into a database, for example, uploading a video to demonstrate to clinical staff how they use their communication devices [43]. However, there is now a striking need to develop high quality and innovative technological interventions to support growing numbers of people with cancer.

Inspection of the International Standard Randomised Controlled Trial Number Register indicates that increasing numbers of clinical trials of technological applications to healthcare are occurring [44]. The register lists twentythree active trials on telehealth, telemedicine or ehealth, including trials in men with prostate cancer and another amongst patients with breast or colorectal cancer [45,46]. Nevertheless, this is surprisingly few randomised trials given the explosion in technological innovation in recent years. It could be that technology is evolving so fast that potential innovative technological interventions become outdated before they can mature sufficiently to be subjected to randomised trials.

\section{Conclusions}

At the current time there are few well conducted randomised trials exploring the role of digital and other technologies in the delivery of structured cancer aftercare. There is, however, some evidence that modern technologies can be used to safely and effectively deliver aspects of structured cancer follow-up as an alternative or adjunct to existing traditional models which require patients to travel to tertiary cancer centres for face to face visits with a cancer specialist. Given the potential gains offered by digital technology in terms of patient convenience and empowerment and reduction of resource use in cancer centres, there is an urgent need for future cancer research to embrace the digital age and seek to integrate various different technologies into innovative and comprehensive models of virtual cancer follow-up. It is essential, however, that such trials employ robust and consistent measures of patient satisfaction and acceptability, clinical safety and cost effectiveness so that powerful evidence on these outcomes can accumulate.

\section{Additional files}

\section{Additional file 1: Search strategy used for embase.}

\section{Competing interests}

All authors have seen and approved the final manuscript. They do not have any competing interests to declare. The authors have full control of all primary data and they agree to allow the journal to review their data if requested.

\section{Authors' contributions}

$\mathrm{RD}, \mathrm{CB}$ and PM designed the review. RD conducted the review assisted by $\mathrm{SH}$. JS participated in updating the review. RD and PM wrote the manuscript with comments on drafts from SH, JS and CB. All authors read and approved the final manuscript.

\section{Acknowledgements}

We wish to acknowledge the assistance of Dr Mel Bickerton, Medical Librarian at the University of Aberdeen, in the design and execution of the search strategy. We also wish to acknowledge the University of Aberdeen who acted as the sponsors for this study. We also acknowledge the support 
of NHS Education for Scotland who funded RD Academic Fellowship, during which this work was conducted. We also acknowledge support to SH from an award made by the RCUK Digital Economy programme to the dot.rural Digital Economy Hub at the University of Aberdeen; award reference: EP/ G066051/1.

\section{Author details}

${ }^{1}$ Wednesbury Malling Health Practice, High Bullen, Wednesbury, West Midlands WS10 7HP, UK. ²Division of Applied Health Science, Centre of Academic Primary Care, University of Aberdeen, Polwarth Building,

Foresterhill, Aberdeen AB25 2ZD, Scotland, UK.

Received: 4 October 2013 Accepted: 17 April 2014

Published: 3 May 2014

\section{References}

1. Brennan ME, Butow P, Marven M, Spillane AJ, Boyle FM: Survivorship care after breast cancer treatment- Experiences and preferences of Australian women. Breast 2011, 20:271-277.

2. Macbride SK, Whyte F: Survivorship and the cancer follow-up clinic. Eur J Cancer Care 1998, 7:47-55.

3. Hall S, Samuel L, Murchie P: Shared follow-up for cancer: developing the model with patients and GPs. Fam Pract 2011, 28:554-564

4. Lewis R, Neal R, Williams N, France B, Hendry M, Russell D, Hughes D, Russell I, Stuart N, Weller D, Wilkinson C: Follow up of cancer in primary care versus secondary care: a systematic review. Br J Gen Pract 2009, 59:e234-e247.

5. Maddams J, Brewster D, Gavin A, Steward J, Elliot J, Utley M, Moller H: Cancer prevalence in the United Kingdom: estimates for 2008. Br J Cancer 2009, 101:541-547.

6. Okera M, Baker NA, Hayward AM, Selva-Nayagam S: Oncology workforce issues: the challenge of the outpatient clinic. Intern Med J 2011, 41:499-503.

7. Green J, Murchie P, Lee AJ: Does place of residence affect the management of cutaneous melanoma? Analysis of a database from Northern Scotland. J Rural Health 2013. doi: 10.1111/jrh.12011.

8. NHS Improvement Rapid Review of current service provision following cancer treatment. Available at http://www.ncsi.org.uk/ (accessed 15th May 2013).

9. Sood S, Mbarika V, Jugoo S, Dookhy R, Doarn CR, Prakash N, Merrell RC: What is telemedicine? A collection of 104 peer-reviewed perspectives and theoretical underpinnings. Telemed J E Health 2007, 13:573-590.

10. McLean S, Protti $D$, Sheikh A: Telehealthcare for long term conditions. BMJ 2011, 342:374-378.

11. Beaver K, Tysver-Robinson D, Campbell M, Twomey M, Williamson S, Hindley A, Susnerwala S, Dunn G, Luker K: Comparing hospital and telephone follow-up after treatment for breast cancer: randomised equivalence trial. BMJ 2009, 338:a3147.

12. Kearney N, McCann L, Norrie J, Taylor L, Gray P, McGee-Lennon M, Sage M, Miller M, Maguire R: Evaluation of a mobile phone-based, advanced symptom management system (ASyMS) in the management of chemotherapy-related toxicity. Support Care Cancer 2009, 17:437-444.

13. Kimman ML, Bloebaum MMF, Dirksen CD, Houben RMA, Lambin P, Boersma $\sqcup$ : Patient satisfaction with nurse-led telephone follow-up after curative treatment for breast cancer. BMC Cancer 2010, 10:174

14. Downs S, Black N: The feasibility of creating a check-list for the assessment of the methodological quality both of randomised and non-randomised studies of health care interventions. J Epidemiol Community Health 1998, 52:377-384.

15. Davison BJ, Degner LF: Feasibility of using a computer-assisted intervention to enhance the way women with breast cancer communicate with their physicians. Cancer Nurs 2002, 25:417-424.

16. Hegel MT, Lyons KD, Hull JG, Kaufman P, Urquhart L, Li Z, Ahles TA: Feasibility study of a randomized controlled trial of a telephone-delivered problem-solving-occupational therapy intervention to reduce participation restrictions in rural breast cancer survivors undergoing chemotherapy. Psychooncology 2011, 20:1092-1101

17. Kimman ML, Dirksen CD, Voogd AC, Falger P, Giijsen BCM, Thuring M, Lenssen A, Van Der Ent F, Verkeyn J, Haekens C, Hupperets P, Nuytinck JKS, Van Riet Y, Brenninkmeijer SJ, Scheijmans LJEE, Kessels A, Lambin P, Boersma L: 2011. Nurse-led telephone follow-up and an educational group programme after breast cancer treatment: Results of a $2 \times 2$ randomised controlled trial. Eur J Cancer 2011, 47:1027-1036.
18. Marcus AC, Garrett KM, Cella D, Wenzel L, Brady MJ, Fairclough D, Pate-Willig M, Barnes D, Emsbo SP, Kluhsman BC, Crane L, Sedlacek S, Flynn PJ: Can telephone counselling post-treatment improve psychosocial outcomes among early stage breast cancer survivors? Psychooncology 2010, 19:923-932.

19. Sandgren AK, McCaul KD: Short-term effects of telephone therapy for breast cancer patients. Health Psychol 2003, 22:310-315.

20. Matthew AG, Currie KL, Irvine J, Ritvo P, Santa Mina D, Jamnicky L, Nam R, Trachtenberg J: Serial personal digital assistant data capture of health-related quality of life: a randomized controlled trial in a prostate cancer clinic. Health Qual Life Outcomes 2007, 5:38.

21. Harrison JD, Young JM, Solomon MJ, Butow PN, Secomb R, Masya L: Randomized pilot evaluation of the supportive care intervention "CONNECT" for people following surgery for colorectal cancer. Dis Colon Rectum 2011, 54:622-631.

22. Sikorskii A, Given CW, Given B, Jeon S, You M: Differential symptom reporting by mode of administration of the assessment: automated voice response system versus a live telephone interview. Med Care 2009, 47:866-874.

23. Sikorskii A, Given CW, Given B, Jeon S, Decker V, Decker D, Champion V, McCorkle R: Symptom management for cancer patients: a trial comparing two multimodal interventions. J Pain Symptom Manage 2007, 34:253-264

24. Yun YH, Lee KS, Kim KW, Park SY, Lee ES, Noh DY, Kim S, Oh JH, Jung SY, Chung KW, Lee YJ, Jeong SY, Park KJ, Shim YM, Zo Jl, Park JW, Kim YA, Shon EJ, Park S: Web-based tailored education program for disease-free cancer survivors with cancer related fatigue: A randomised controlled trial. J Clin Oncol 2012, 30:1296-1303.

25. Beaver K, Hollingworth W, McDonald R, Dunn G, Tysver-Robinson D, Thomson L, Hindley AC, Susnerwala SS, Luker K: Economic evaluation of a randomized clinical trial of hospital versus telephone follow-up after treatment for breast cancer. Br J Surg 2009, 96:1406-1415.

26. Kroenke K, Theobald D, Wu J, Norton K, Morrison G, Carpenter J, Tu W: Effect of telecare management on pain and depression in patients with cancer: a randomized trial. JAMA 2010, 304:163-171.

27. Kimman ML, Dirksen CD, Voogd AC, Falger $P$, Gijsen BC, Thuring M, Lenssen A, van der Ent F, Verkeyn J, Haekens C, Hupperets P, Nuytinck JK, van Riet $Y$, Brenninkmeijer SJ, Scheijmans LJ, Kessels A, Lambin P, Boersma LJ: Economic evaluation of four follow-up strategies after curative treatment for breast cancer: results of an RCT. Eur J Cancer 2011, 47:1175-1185.

28. Van den Brink JL, Moorman PW, de Boer M, Hop WCJ, Pruyn JFA, Verwoerd CDA, van Bemmel JH: Impact on quality of life of a telemedicine system supporting head and neck cancer patients: a controlled trial during the postoperative period at home. J Am Med Inform Assoc 2006, 14:198-205.

29. Cox K, Wilson E: Follow-up for people with cancer: nurse-led services and telephone interventions. J Adv Nurs 2003, 43:51-61.

30. Murchie P, Nicolson MC, Hannaford PC, Raja EA, Lee AJ, Campbell NC Patient satisfaction with GP-led melanoma follow up: a randomised controlled trial. Br J Cancer 2010, 102:1447-1455.

31. Hazin R, Qaddoumi I: Teleoncology: current and future applications for improving cancer care globally. Lancet Oncol 2010, 11:204-210.

32. Hede K: Teleoncology gaining acceptance with physicians, patients. J Natl Cancer Inst 2010, 102:1531-1533.

33. Palkhivala A: Canada develops models of teleoncology. $J$ Natl Cancer Inst 2011, 103:1566-1567.

34. Sudhamony S, Nandakumar K, Binu PJ, Issac NS: Telemedicine and tele-health services for cancer-care delivery in India. IET Commun 2008, 2:231-236.

35. Allen A, Hayes J: Patient satisfaction with teleoncology: a pilot study. Telemed J 1995, 1:41-46.

36. Ricke J, Bartelink H: Telemedicine and its impact on cancer management. Eur J Cancer 2000, 36:826-833.

37. Coelho JJ, Arnold A, Nayler J, Tischkowitz M, MacKay J: An assessment of the efficacy of cancer genetic counselling using real-time videoconferencing technology (telemedicine) compared to face-to-face consultations. Eur J Cancer 2005, 41:2257-2261.

38. Weinstein RS, López AM, Barker GP, Krupinski EA, Descour MR, Scott KM Richter LC, Beinar SJ, Holcoms MJ, Bartels PH, McNeely RA, Bhattacharyya AK: The innovative bundling of teleradiology, telepathology, and teleoncology services. IBM Syst J 2007, 46:69-84

39. Ekeland AG, Bowes A, Flottrop S: Effectiveness of telemedicine: a systematic review of reviews. Int J Med Inform 2010, 79:736-771. 
40. Franklin VL1, Waller A, Pagliari C, Greene SA: A randomized controlled trial of Sweet Talk, a text-messaging system to support young people with diabetes. Diabet Med 2006, 23:1332-1338.

41. Bennett DA, Emberson JR: Text messaging in smoking cessation: the txt2stop trial. Lancet 2011, 378:6-7.

42. Kaiser Permanente (2012) My health manager; 2012. https://healthy. kaiserpermanente.org/health/care/consumer/my-health-manager. (Accessed 20 Feb 2014).

43. Prior S: Involving adults with severe speech and physical impairments in the design of CHAMPION. In ACM SIGCHI conference on human factors in computing systems. Atlanta, USA: ACM; 2010.

44. International Standard Randomised Controlled Trial Number Register; 2014 http://www.controlled-trials.com/isrctn/search.html (accessed 20th February 2014).

45. Eysenbach G: Impact of Internet Instructions on Men with Prostate Cancer. ISRCTN10001875. doi:10.1186/ISRCTN10001875. http://www.controlled-trials. com/isrctn/search.html (accessed 20th February 2014).

46. Orruño E: Evaluation of an e-health intervention for cancer patients'support. ISRCTN00735390. doi:10.1186/ISRCTN00735390. http://www.controlled-trials. com/isrctn/search.html (accessed 20th February 2014).

doi:10.1186/1471-2407-14-311

Cite this article as: Dickinson et al: Using technology to deliver cancer

follow-up: a systematic review. BMC Cancer 2014 14:311.

\section{Submit your next manuscript to BioMed Central and take full advantage of:}

- Convenient online submission

- Thorough peer review

- No space constraints or color figure charges

- Immediate publication on acceptance

- Inclusion in PubMed, CAS, Scopus and Google Scholar

- Research which is freely available for redistribution 